\title{
7T MRI super-resolution with Generative Adversarial Network
}

\author{
Huy Do ${ }^{1,2}$, Pascal Bourdon ${ }^{1,2}$, David Helbert ${ }^{1,2}$, Mathieu Naudin ${ }^{2,3}$, Remy Guillevin ${ }^{2,3,4}$ \\ ${ }^{1}$ Xlim-ASALI, CNRS U-7252, University of Poitiers, France \\ 2 I3M Common Laboratory CNRS-Siemens, University and Hospital of Poitiers, France \\ ${ }^{3}$ Poitiers University Hospital, CHU; Poitiers, France \\ ${ }^{4}$ DACTIM-MIS/LMA Laboratory University of Poitiers, UMR CNRS 7348; Poitiers, France
}

\section{Abstract}

The high-resolution magnetic resonance image (MRI) provides detailed anatomical information critical for clinical application diagnosis. However, high-resolution MRI typically comes at the cost of long scan time, small spatial coverage, and low signal-to-noise ratio. The benefits of the convolutional neural network (CNN) can be applied to solve the super-resolution task to recover high-resolution generic images from low-resolution inputs. Additionally, recent studies have shown the potential to use the generative advertising network (GAN) to generate high-quality super-resolution MRIs using learned image priors. Moreover, existing approaches require paired MRI images as training data, which is difficult to obtain with existing datasets when the alignment between high and low-resolution images has to be implemented manually.

This paper implements two different GAN-based models to handle the super-resolution: Enhanced superresolution GAN (ESRGAN) and CycleGAN. Different from the generic model, the architecture of CycleGAN is modified to solve the super-resolution on unpaired MRI data, and the ESRGAN is implemented as a reference to compare GAN-based methods performance. The results of GAN-based models provide generated highresolution images with rich textures compared to the ground-truth. Moreover, results from experiments are performed on both $3 \mathrm{~T}$ and $7 \mathrm{~T}$ MRI images in recovering different scales of resolution.

\section{Introduction}

In recent years, there has been a huge increase of computer vision research leading to an important proportion of information related to this field. Image superresolution (SR) is an exploitable image processing field that refers to recovering high-resolution (HR) images with rich details from given low-resolution (LR) images.

At the present time, several deep learning-based methods have been proposed to improve the quality of images. Convolutional neural networks $(\mathrm{CNN})$ can model various complex structures and utilize large quantities of training data. After completing the training phase, no additional process is required to produce output images; thus, it does not cost time for the sub-sequence process. Recently, many CNN-based methods have out- performed in the field of SR. Dong et at.[1] proposed the SRCNN, which learns an end-to-end mapping from LR image to HR images. Since the work of Dong et al. [1], different CNN based super-resolution approaches have been implemented.

By improving the network architectures and training process, CNN-based SR has achieved significant success on both objective (peak signal-to-noise ratio - PSNR) $[2,3,4]$ and subjective (human visual quality assessment tests) $[3,5]$ criteria. A deep neural network with large capacity can improve the performance in general [6]. However, it also increases the computation cost of the training phase. With different techniques such as skip connection [7], embedding [8] or normalization [9], reducing computation time for a effective training now is possible. Following the study of Kim et al. [4], a deep network using advanced techniques to increase network depth could ease the difficulty of training networks, and the structure of the neural network is the key to obtain the high quality of SR outputs.

Tong et al. [10] have proposed the densely connected network (SRDenseNet) to solve SR tasks, using several hierarchical features in different layers into the final construction layer. Sub-sequence layers can effectively utilize extracted feature information from convolutional layers. It is a new measure to preserve the features of different levels extracted from different convolutional layers in the network. It retains the ground truth image features to a greater extent, so the dense connection can effectively improve image reconstruction quality. SRDenseNet has significantly improved performance over the model using multi-level features, indicating that level fusion is indeed beneficial for SR problems. Besides, since ResNet has been published by He et al. [7], it has proved its efficiency in reducing training computation time and benefit in SR fields [11]. Since their introduction in [12], generative adversarial networks (GAN) have become a trend to solve many different computer vision problems. The generic GAN model includes a generator and a discriminator. While the generator is trained to learn a mapping from source images in a domain to target images in another domain, the discriminator distinguishes and targets generated images with binary labels. Once well trained, GAN can perform on high-dimensional target images.

Following the progress of neural networks applied in natural images, medical image analysis is one of several applications that is benefited from the improvement of 
SR approaches. Further research has been proposed to improve medical image quality, such as computed tomography (CT) or Magnetic Resonance Imaging (MRI).

MRI is widely used in medical imaging because of its non-invasive assessment of the body's anatomy and physiology in both health and disease while providing the best contrast resolution on soft tissues. However, MRI images are normally acquired with a finite resolution limited by the signal-to-noise ratio (SNR), hardware, or time limitation. In the clinical and research centers, usually high-resolution and high-contrast MRI is preferred because it provides critical structural details with a smaller voxel size. Therefore, the request for image quality with sufficient details is fast increasing in medical imaging. In recent years, the ultra-highfield MR 7T scanners were introduced, which allow high-resolution MRI scanning with many advantages compared to routine MRI, such as $3 \mathrm{~T}$ MRI or $1.5 \mathrm{~T}$. The 7T MRI scanner can provide higher resolution images while maintaining a similar signal-to-noise ratio (SNR), which has a linear relation to the magnetic field in general for MRI scanning.

Recently, many studies have been proposed for medical image analysis, especially super-resolution. the variety of methods stretches from statistical method such as interpolation [13, 14], dictionary mapping [15, 16], self-learning [17] to automatically techniques such as CNN based-method [18, 19] or hybrid methods [20]. However, most existing approaches are implemented on private datasets because algorithms require paired datasets for training. Such datasets usually come from collaborating hospitals. However, these are not large enough due to the tedious and time-consuming task of paired data retrieval. As an example, to obtain paired $3 \mathrm{~T}$ and $7 \mathrm{~T}$ MRI, patients must take the test on the same day, and later, images have to be aligned manually. Besides, there is also the difficult to publish due to security issues.

For super-resolution task, a paired dataset contains both low and high-resolution MRI, which is not easy to obtain. There is no available scanner to produce HR MRI, which generally have $\mathrm{HD}$ or $2 \mathrm{~K}$ resolution. All the pre-process of super-resolution has to be prepared manually with a very complicated process of alignment. At this moment, there is no public dataset with paired MRI.

This paper implements two different types of GANbased models to solve SR on MRI images: the Enhanced Super-Resolution GAN (ESRGAN) and CycleGAN. The advantage of the approach is that image pairing is not required for the training dataset. Therefore they can be executed on several publicly available MRI datasets, thus overcoming the limitations explained earlier.

CycleGAN is known as a very popular method for image-to-image translation. However, the superresolution problem requires some special characteristics due to the difference between input and output. With some modifications to network architecture, we make CycleGAN fit with the SR problem. The generator is modified with several different building blocks from the generic CycleGAN to automatically solve up-sample and down-sample images by applying skip connection techniques. In term of ESRGAN [21], it is an improved version of SRGAN [3]. With these special modifications of network architecture, ESRGAN has shown excellent performance in SR fields. To our knowledge there is no ESRGAN implementation for medical images at the present time, therefore we decide to explore ESRGAN performance in MRI super-resolution. It also can be used as a reference to compare with CycleGAN performance later.

In the next section, we introduce background knowledge and concepts of image super-resolution with different CNN-based methods and techniques. Then we discuss the details of network architectures used in our experiments.

\section{Related work}

\section{$2.1 \quad$ Image super-resolution}

Before deep learning-based approaches achieved stateof-the-art performance, SR techniques mostly relied on statistical analysis, interpolation, edge-preservation, and sparse dictionary learning[22]. Since the study of Dong et al. [1], CNNs have been increasingly popular to solve the SR problem. The SRCNN can handle feature extraction, feature space building and image reconstruction together in an end-to-end training. Later, many follow-up approaches with the improvement of network structures have been inspired by the SRCNN $[4,10,23]$.

\subsection{Residual, dense blocks and skip connection}

The performance of deep learning methods is fast improving. In contrast, these models are significantly increasing model size, wherein the depth of modes becomes a practical problem. Moreover, when the network is too deep, gradient disappearance and gradient explosion issues are declared [24]. Although these gradient issues can be solved by data regulation and batch normalization, it can lead to model performance degradation. To address this problem, ResNet proposed by He et al. [7] introduced residual learning, where the output of the previous convolutional layer connected to the next for smoother information flows through a short cut. The detail of residual blocks is shown in Figure 1. The shortcut connection neither increases the number of network parameters nor the computational complexity of algorithms.

DenseNet [25] proposed a connectivity pattern to improve the flow of feature information by concatenating information of previous layers. The network is more efficient and outperforms ResNet with fewer parameters. In addition, recent studies in image super-resolution show that removing BN layers in residual blocks [21] and dense blocks [2] can reduce computational cost, memory usage, and boost model performance. The 
flow of the gradient is unobstructed due to the direct link between layers. SRDenseNet[10] is a version of DenseNet to solve the SR problems.

Additionally, Zhang et al. [26] introduced Residual Dense Network (RDN), a combination of DenseNet and ResNet to solve the image super-resolution task by using residual dense blocks (RDB), which contain several dense connected layers, a local feature fusion, and the residual learning as the final layer. Figure 1 illustrates the design of all blocks in different superresolution models with the removing of BN layers .

\subsection{Generative adversarial network and GAN-based Super-resolution}

Since Goodfellow et al. citegoodfellow2014generative have proposed the generative adversarial networks (GAN) model, many follow-up studies of GAN and its variations have been applied in several computer vision tasks. In general, GAN has proved its efficiency to achieve state-of-the-art performance in the image synthesis field. Recently, GAN has also been applied in the super-resolution field. SRGAN [3] and ESRGAN [21] have been successfully applied to solve SR problems for color images. Besides, CycleGAN [27] proposed by Zhu et al. is an image-to-image translation model for learning to translate an image from a source domain to a target domain in the absence of paired examples. In general, CycleGAN has been applied in image synthesis and image translation domain. By applying the benefits of skip connection techniques and CNN based methods, we can also apply CycleGAN to solve SR problems. The advantage of both methods is that they do not require paired image datasets, which is challenging to achieve HR MRI image due to the limit of devices and manual process.

Most previous super-resolution methods aim to optimize HR image reconstruction by minimizing the voxel-wise difference between original and generated images. However, a drawback that merely cares about local pixel-wise differences leads to extreme difficulty in restoring important small details. In contrast, if global perceptual constraints can be taken into account, the SR model is guided by both local intensity information and patch-wise perceptual information, probably leading to a better and sharper SR reconstruction [3].

With benefits from the GAN framework of Goodfellow et al. [12] for its unsupervised-learning potential of capturing perceptually important image features, Ledig et al. [3] proposed the SRGAN to handle the superresolution issue. The principle of GAN relies on two components: a generator and a discriminator. While the generator generates fake images as real as possible, the discriminator distinguishes and evaluates to improve generated image accuracy. In the end, the discriminator can separate real and generated image, while the generator can produce realistic-looking images. Next, the perceptual loss is defined in the GAN model [12] and extended in SRGAN. SRGAN defines the perceptual loss on the activation layers of a pretrained deep network, where the distance between two activated features is minimized.

Several techniques have been implemented to provide different architectures of building units to transform LR into HR image and reduce computation cost during the training phase. Residual blocks (RB) from ResNet of He et al. [7] and Dense block (DB) from DenseNet of Huang et al. [25] are the most popular architecture use in SR tasks. Based on different models, these building blocks can be combined or modified. In SRGAN, residual blocks have been used for the generator.

\section{ESRGAN}

Improved from SRGAN, ESRGAN proposed by Wang et al. [21] aims to increase the quality of HR images. While SRGAN uses residual blocks as basic blocks, ESRGAN uses Residual-in-Residual Dense blocks (RRDB) without Batch Normalization (BN) layers as building units. As shown in Figure 1, RRDB is a combination of multi-level residual blocks and dense block connection.

Recent studies have shown that removing BN layers can reduce computational cost, memory usage, and boost model performance [2]. Although BN layers use mean and variance computations to normalize the features during training and later during testing, BN layers tend to produce unpleasant artifacts and limit generalization ability when the difference between training and testing set is significant[21].

The addition of layer and connections can improve model performance [26, 28], RRDB exploits deeper and more complex connections than the residual blocks of SRGAN. Meanwhile, the general high-architecture of the ESRGAN model is kept as SRGAN. The details of the generators with the additions of RRDB are shown in Figure 2.

The discriminator of ESRGAN is also improved from SRGAN, based on the relativistic GAN [29]. Instead of estimating the probability of an input being real or natural, the relativistic discriminator calculates the probability of a real image to be relatively more realistic than a fake image [21].

In terms of perceptual loss, ESRGAN uses features before the activation layers, which helps overcoming the drawbacks of the original design. The perceptual loss in SRGAN can cause inconsistent reconstructed brightness compared with the ground-truth image or the sparsely activate of features when deep network is very deep [21]. The architecture of ESRGAN is implemented from the original as a reference to compare performance of GAN-based model.

\section{CycleGAN}

The core problem of the SR task in the real world is the lack of paired datasets. Zhu et al. [27] introduced CycleGAN - an image-to-image translation framework using unpaired data. Later, it has inspired many following studies in different computer vision tasks, and potential for SR. CycleGAN aims to translate input images from a class into another class without the requirement of 


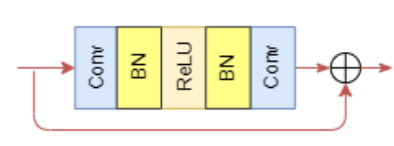

(i) Residual Blocks

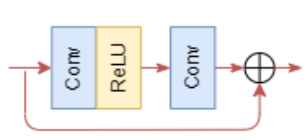

(ii) Residual Block w/o BN

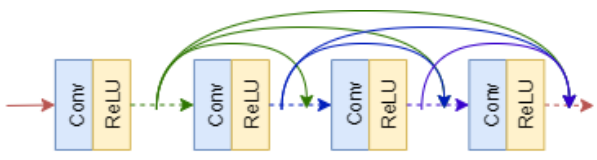

(iii) Dense Block w/o BN

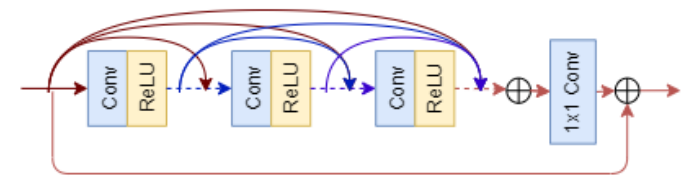

(iv) Residual Dense Blocks

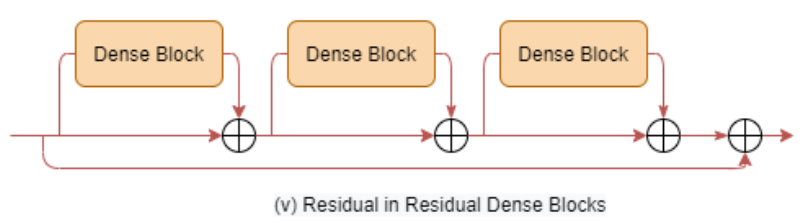

Figure 1: Architecture of building blocks in network. (i) Residual blocks in SRGAN[3] (ii) Residual blocks without BN in [2] (iii) Dense blocks in SRDenseNet[10] (iv) Residual Dense Blocks from (ii) and (iii) in [26], (v) Residual-in-residual dense blocks in ESRGAN[21]

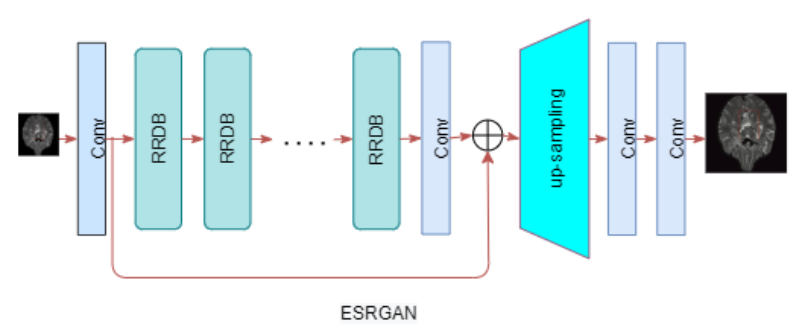

Figure 2: Network architecture of ESRGAN generator [21].

paired images during training. However, different from traditional image translation, which assumes input and output images have the same size, SR requires output images larger than the inputs, making it very difficult to apply CycleGAN directly.

A recent study of Zhang et al. [26] has introduced the Residual Dense Network (RDN), which provides an exemplary architecture of basic blocks. Based on residual blocks and dense blocks, RDN proposed a Residual Dense Blocks (RDB) to handle super-resolution tasks. Like the RRDB, RDB also removes BN layers to reduce computational time, memory usage, and to speed up the training process. With the addition of the basic block, the network also introduces contiguous memory [26], which allows direct access of information between each block layer. The model also improves the preservation of information inside the network using local and global feature learning which also combine the low and high-level features.

Using the RDN, we focus on the generator architecture of CycleGAN to adapt to super-resolution problems. In the super-resolution CycleGAN, the generator contains several RDB for feature extractions with local and global residual learning to keep information convection. Next, local and global features are stacked for up-sampling or down-sampling to produce output. The details of the generators with the additions of RDB is shown on Figure 3.

A generic CycleGAN model can perform translations between two classes in both directions. Therefore we build two different generators to up-sample from LR to

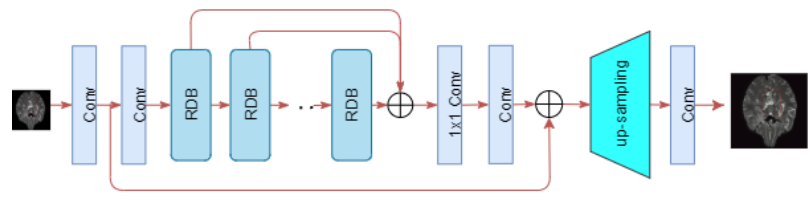

Figure 3: Network architecture of CycleGAN generator. The generator contains several RDBs with local residual learning structure to improve information flow. At the higher architecture, the global residual learning structure use multi-level features to synthesize information in LR before up-sampling.

HR and down-sample from HR to LR simultaneously. The architecture of the discriminator is kept to evaluate the performance of generators.

The cycle architecture or CycleGAN is kept identical to the original model [27]. The generator should eventually be able to trick the discriminator about the authenticity of its generated images. This can be done if the recommendation factor of the discriminator for generated images is as close to 1 as possible. The content of cycle consistency and adversarial loss - the outstanding properties of CycleGAN - is kept. The adversarial loss of CycleGAN is calculated based on the MSE loss between the real and fake images of two classes. To handle the generating of non-related output from input, the cycle loss consistency is applied. In detail, when an image is generated, the second generator will convert it back to the original class. Then, the cycle consistency is calculated by the average of L1 loss between the real input and the cyclic output. The difference between the original image and the cyclic image should be as small as possible.

The discriminator must be trained such that recommendation factor for images from the first category be as close to 1 as possible, and vice versa for the second discriminator. So the first discriminator would like to minimize the loss between the generated and the real ones, and the same goes for the second category as well. Since discrimination should be able to distinguish between generated and original images, it should also 
be predicting 0 for images produced by the generator.

\section{$5 \quad$ Experiment and Results}

\section{Dataset}

At this moment, there is no benchmark MRI dataset for the super-resolution task. Current MRI scanners are not available to produce high-resolution MRI, which generally have HD or $2 \mathrm{~K}$ resolution. Obtaining highresolution MRIs requires them to be processed manually through a complex alignment system.

As mentioned in the previous section, we aim to implement methods that does not require paired images as training data. In this project, we used the BraTS2018 [30] dataset - a public dataset containing 3T MRI images with different types of sequences. BraTS is a very popular dataset used in the segmentation and classification domain. The variety of the dataset is confirmed when samples were acquired with different clinical protocols and various scanners from multiple institutions. The field of view is $155 \times 240 \times 240$, wherein the slice thickness is $1 \mathrm{~mm}$. The size of the original image is kept at a 1:1 ratio when the output is $240 \times 240$ pixels.

Within the project scope, we also want to explore the performance of methods on different types of MRI. $7 \mathrm{~T}$ MRI has proved its function in medical image analysis. However, there is no $7 \mathrm{~T}$ dataset available at this moment. With the support of the 7T system from Siemens Healthineers at Poitiers University Hospital, we can build a dataset containing several brain 3T and 7T MRI samples with high-quality. We aim to use that dataset to explore super-resolution performance. Different from 3T, 7T technology provides MRI images with higher quality and deeper voxel spacing between slices. The field of view is $255 \times 320 \times 320$, wherein the slice thickness is $0.7 \mathrm{~mm}$. The original image's size is also kept at a 1:1 ratio when the output is $320 \times 320$ pixels.

\section{Training setting}

The training process is implemented on BraTS2018, which contains only $3 \mathrm{~T}$ MRI. Later, the testing set of BraTS and 7T MRI dataset is used on the testing phase to evaluate the performance of the superresolution model. Due to the lack of high-resolution (HD or $2 \mathrm{~K}$ ) MRI, training images in the dataset are degraded into lower-resolution to simulate LR images with scale factors. All image resolution changes are implemented during the pre-processing step without modifying original data during the training phase.

The training of both ESRGAN and CycleGAN is executed on patches to ensure the diversity of data. Besides, we also want to reduce the computational cost of the model during the training process. The complexity of GAN-based models is very considerable, with millions of parameters. This number will increase along with the increase of model depth or the size of the input. For each training batch, images are randomly extracted into 16 patches on LR images corresponding to a size of $64 \times 64$ on HR images.
For CycleGAN, we keep the content of adversarial loss and cycle consistency loss. The generator is modified to contain residual dense blocks. In CycleGAN, due to the network architecture, two generators are built to up-sample and down-sample images. In the downsample generator, there are two options to down-sample images with convolutional layers or pooling layers. The learning rate is initialized to 0.0005 and decay starts after every 100 iterations. The ADAM optimizer is used to update network weights based on training data.

For ESRGAN, we have implemented the model following the original paper to use it as a reference for the GAN-based method reference. The generator contains several RRDB. Different from CycleGAN, a single ESRGAN model only can implement the upsample or down-sample images. The learning rate is initialized to 0.0002 and decay starts after the fifth epoch. The ADAM optimizer is also used to update network weights. The first 100 batches are to "warm-up" the training phase, which uses only pixel-wise loss.

\section{Results}

Benefiting from these improvements, results of GANbased models provide generated high-resolution images with rich textures that compare to ground-truth data. Results from our experiments are performed on both $3 \mathrm{~T}$ and 7T MRI images to reconstruct different resolution levels. Figure 4 shows the result of ESRGAN and CycleGAN on 3T and 7T MRI images with different types of sequences.

\begin{tabular}{|c|c|c|c|c|c|c|}
\hline & \multicolumn{2}{|c|}{ Bicubic } & \multicolumn{2}{c|}{ ESRGAN } & \multicolumn{2}{c|}{ CycleGAN } \\
\hline & PSNR & SSIM & PSNR & SSIM & PSNR & SSIM \\
\hline 3T MRI & 27.26 & 0.52 & 35.85 & 0.88 & $\mathbf{3 6 . 7 6}$ & $\mathbf{0 . 9 2}$ \\
\hline 7T MRI & 25.45 & 0.51 & 31.85 & 0.59 & $\mathbf{3 6 . 7 9}$ & $\mathbf{0 . 9 1}$ \\
\hline
\end{tabular}

Table 1: Average value of PSNR (dB) and SSIM for scale factor $\times 4$ on $3 \mathrm{~T}$ and $7 \mathrm{~T}$ MRI data

Table 1 shows average distortion and fidelity values obtained on the test set by GAN-based methods. We have also included simple bicubic interpolation to use it as a reference for quality. Image quality measurement in terms of PSNR and SSIM shows that CycleGAN methods can achieve relatively low distortion at the $\times 4$ scale factor, while ESRGAN is still limited. We consider that the CycleGAN has better and more stable performance compared to ESRGAN.

As shown on Figure 4 and Table 1, both ESRGAN and CycleGAN provide super-resolution 3T MRI images with rich textures. The performance of CycleGAN outperforms that of ESRGAN especially on 7T MRI data. CycleGAN SSIM scores exceed 0.9, thus ensuring a better fidelity of image structures such as contours or fine details compared to the original images. The generated images of ESRGAN contains noise, while the CycleGAN images are relatively stable. 


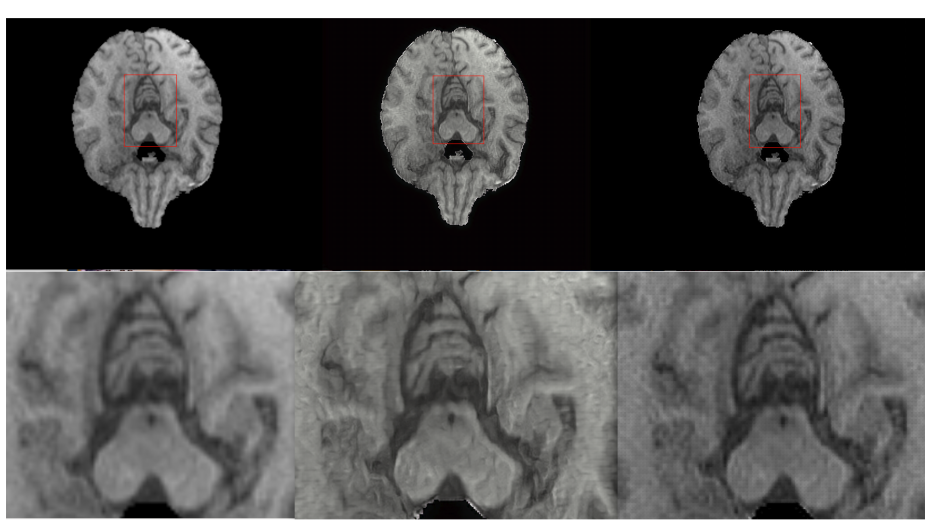

(a) $3 \mathrm{~T}$ T1 MRI superresolution

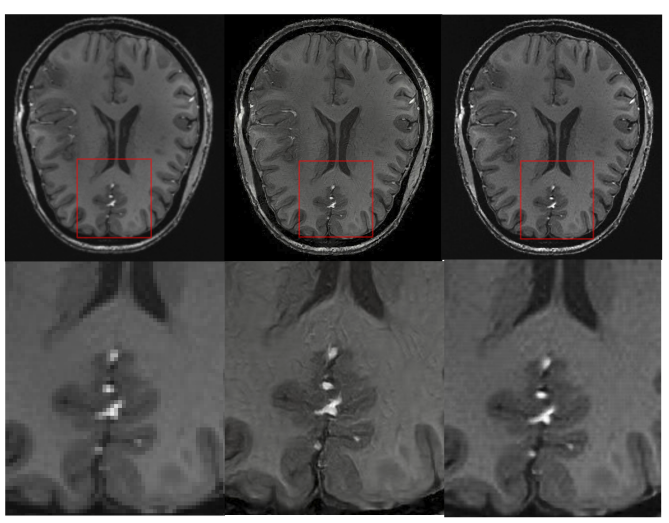

(b) 7T T1 MRI super-resolution

Figure 4: Super-resolution on MRI images. From top to bottom: full-size and zoom image. From left to right: original image, ESRGAN image, CycleGAN image

\section{Conclusion}

Until now, the existing methodologies in the field of MRI SR required paired low and high-resolution MRI images for training, which are difficult to obtain. Due to the advantage of the GAN-based methods, we can use the BraTS 2018 dataset as unpaired training data. We also test the performance of models on $7 \mathrm{~T}$ MRI data.

The CycleGAN model is a prevalent method known for image synthesis. With the advantage of self-learning between two different classes, it can be used for superresolution tasks. By the modification of network architecture, we use CycleGAN to match the problem of SR. To have a reference to compare with CycleGAN, we also implemented ESRGAN - a popular method in the super-resolution task which has no implementation for medical images as far as we know.

Experimental results show that the performance of CycleGAN on BraTS 2018 and on our 7T dataset is better and more stable than that of the ESRGAN model. In further works, we intend to validate our results with subjective tests made performed on a population of radiologists trained for $7 \mathrm{~T}$ analysis.

\section{References}

[1] Chao Dong, Chen Change Loy, Kaiming He, and Xiaoou Tang, "Learning a deep convolutional network for image super-resolution," in European conference on computer vision. Springer, 2014, pp. 184-199.

[2] Bee Lim, Sanghyun Son, Heewon Kim, Seungjun Nah, and Kyoung Mu Lee, "Enhanced deep residual networks for single image super-resolution," in Proceedings of the IEEE conference on computer vision and pattern recognition workshops, 2017, pp. 136-144.

[3] Christian Ledig, Lucas Theis, Ferenc Huszár, Jose Caballero, Andrew Cunningham, Alejandro
Acosta, Andrew Aitken, Alykhan Tejani, Johannes Totz, Zehan Wang, et al., "Photo-realistic single image super-resolution using a generative adversarial network," in Proceedings of the IEEE conference on computer vision and pattern recognition, 2017, pp. 4681-4690.

[4] Jiwon Kim, Jung Kwon Lee, and Kyoung Mu Lee, "Accurate image super-resolution using very deep convolutional networks," in Proceedings of the IEEE conference on computer vision and pattern recognition, 2016, pp. 1646-1654.

[5] Mehdi SM Sajjadi, Bernhard Scholkopf, and Michael Hirsch, "Enhancenet: Single image superresolution through automated texture synthesis," in Proceedings of the IEEE International Conference on Computer Vision, 2017, pp. 4491-4500.

[6] Shizhao Sun, Wei Chen, Liwei Wang, Xiaoguang Liu, and Tie-Yan Liu, "On the depth of deep neural networks: A theoretical view," in Thirtieth AAAI Conference on Artificial Intelligence, 2016.

[7] Kaiming He, Xiangyu Zhang, Shaoqing Ren, and Jian Sun, "Deep residual learning for image recognition," in Proceedings of the IEEE conference on computer vision and pattern recognition, 2016, pp. 770-778.

[8] Christian Szegedy, Sergey Ioffe, Vincent Vanhoucke, and Alexander A Alemi, "Inception-v4, inception-resnet and the impact of residual connections on learning," in Thirty-first AAAI conference on artificial intelligence, 2017.

[9] Sergey Ioffe and Christian Szegedy, "Batch normalization: Accelerating deep network training by reducing internal covariate shift," arXiv preprint arXiv:1502.03167, 2015.

[10] Tong Tong, Gen Li, Xiejie Liu, and Qinquan Gao, "Image super-resolution using dense skip connections," in Proceedings of the IEEE International 
Conference on Computer Vision, 2017, pp. 47994807.

[11] Sam Gross and Michael Wilber, "Training and investigating residual nets," Facebook AI Research, vol. $6,2016$.

[12] Ian Goodfellow, Jean Pouget-Abadie, Mehdi Mirza, Bing Xu, David Warde-Farley, Sherjil Ozair, Aaron Courville, and Yoshua Bengio, "Generative adversarial nets," in Advances in neural information processing systems, 2014, pp. 2672-2680.

[13] Thomas Martin Lehmann, Claudia Gonner, and Klaus Spitzer, "Survey: Interpolation methods in medical image processing," IEEE transactions on medical imaging, vol. 18, no. 11, pp. 1049-1075, 1999.

[14] Philippe Thévenaz, Thierry Blu, and Michael Unser, "Interpolation revisited [medical images application]," IEEE Transactions on medical imaging, vol. 19, no. 7, pp. 739-758, 2000.

[15] Yinghua Li, Bin Song, Jie Guo, Xiaojiang Du, and Mohsen Guizani, "Super-resolution of brain MRI images using overcomplete dictionaries and nonlocal similarity," IEEE Access, vol. 7, pp. 25897 25907, 2019.

[16] Khosro Bahrami, Feng Shi, Islem Rekik, Yaozong Gao, and Dinggang Shen, "7T-guided superresolution of 3T MRI," Medical physics, vol. 44, no. 5, pp. 1661-1677, 2017.

[17] José V Manjón, Pierrick Coupé, Antonio Buades, D Louis Collins, and Montserrat Robles, "MRI superresolution using self-similarity and image priors," International journal of biomedical imaging, vol. 2010, 2010.

[18] Yuhua Chen, Yibin Xie, Zhengwei Zhou, Feng Shi, Anthony G Christodoulou, and Debiao Li, "Brain MRI super resolution using 3d deep densely connected neural networks," in 2018 IEEE 15th International Symposium on Biomedical Imaging (ISBI 2018). IEEE, 2018, pp. 739-742.

[19] Wei Zeng, Jie Peng, Shanshan Wang, and Qiegen Liu, "A comparative study of CNN-based superresolution methods in MRI reconstruction and its beyond," Signal Processing: Image Communication, vol. 81, pp. 115701, 2020.

[20] Liangqiong Qu, Yongqin Zhang, Shuai Wang, PewThian Yap, and Dinggang Shen, "Synthesized 7T MRI from 3T MRI via deep learning in spatial and wavelet domains," Medical Image Analysis, vol. 62, pp. 101663, 2020.

[21] Xintao Wang, Ke Yu, Shixiang Wu, Jinjin Gu, Yihao Liu, Chao Dong, Yu Qiao, and Chen Change Loy, "Esrgan: Enhanced super-resolution generative adversarial networks," in Proceedings of the European Conference on Computer Vision (ECCV), 2018, pp. 0-0.
[22] Chih-Yuan Yang, Chao Ma, and Ming-Hsuan Yang, "Single-image super-resolution: A benchmark," in European Conference on Computer Vision. Springer, 2014, pp. 372-386.

[23] Ying Tai, Jian Yang, and Xiaoming Liu, "Image super-resolution via deep recursive residual network," in Proceedings of the IEEE conference on computer vision and pattern recognition, 2017, pp. 3147-3155.

[24] Karen Simonyan and Andrew Zisserman, "Very deep convolutional networks for large-scale image recognition," arXiv preprint arXiv:1409.1556, 2014.

[25] Gao Huang, Zhuang Liu, Laurens Van Der Maaten, and Kilian Q Weinberger, "Densely connected convolutional networks," in Proceedings of the IEEE conference on computer vision and pattern recognition, 2017, pp. 4700-4708.

[26] Yulun Zhang, Yapeng Tian, Yu Kong, Bineng Zhong, and Yun Fu, "Residual dense network for image super-resolution," in Proceedings of the IEEE conference on computer vision and pattern recognition, 2018, pp. 2472-2481.

[27] Jun-Yan Zhu, Taesung Park, Phillip Isola, and Alexei A Efros, "Unpaired image-to-image translation using cycle-consistent adversarial networks," in Proceedings of the IEEE international conference on computer vision, 2017, pp. 2223-2232.

[28] Yulun Zhang, Kunpeng Li, Kai Li, Lichen Wang, Bineng Zhong, and Yun Fu, "Image superresolution using very deep residual channel attention networks," in Proceedings of the European Conference on Computer Vision (ECCV), 2018, pp. $286-301$.

[29] Alexia Jolicoeur-Martineau, "The relativistic discriminator: a key element missing from standard gan," arXiv preprint arXiv:1807.00734, 2018.

[30] Bjoern H Menze, Andras Jakab, Stefan Bauer, Jayashree Kalpathy-Cramer, Keyvan Farahani, Justin Kirby, Yuliya Burren, Nicole Porz, Johannes Slotboom, Roland Wiest, et al., "The multimodal brain tumor image segmentation benchmark (brats)," IEEE transactions on medical imaging, vol. 34, no. 10, pp. 1993-2024, 2014. 